\title{
Anti-osteoarthritic effects of ChondroT in a rat model of collagenase-induced osteoarthritis
}

Jiwon Jeong ${ }^{1}$, Kiljoon Bae ${ }^{1}$, Sun-Gil Kim² ${ }^{2}$, Dongwook Kwak², Young-Joo Moon³ , Chan-Hun Choi², Young-Ran Kim ${ }^{4}$ Chang-Su $\mathrm{Na}^{2^{*}}$ and Seon-Jong Kim ${ }^{1 *}$

\begin{abstract}
Background: Previously, we reported that ChondorT showed significant anti-arthritis and anti-inflammatory effects. ChondroT, a new herbal medication, consists of the water extracts of Osterici Radix, Lonicerae Folium, Angelicae Gigantis Radix, Clematidis Radix, and Phellodendri Cortex (6:4:4:4:3). The objective of this study was to investigate the effects of ChondroT in collagenase-induced osteoarthritis rat model.

Methods: Osteoarthritis was induced by the injection of collagenase into the right knee joint cavity of rats. The samples were divided into seven groups [intact $(n=6)$, control $(n=6)$, indomethacin $(n=6)$, Joins tab $(n=6)$, ChondroT50 $(n=6)$, ChondroT100 $(n=6)$, and ChondroT200 $(n=6)]$. The control group was administered normal saline, indomethacin group was administered indomethacin ( $2 \mathrm{mg} / \mathrm{kg}$ ), and Joins tab group was administered Joins Tab (20 mg/kg). The ChondroT50, ChondroT100, and ChondroT200 groups were administered 50, 100, and $200 \mathrm{mg} / \mathrm{kg}$ of ChondroT, respectively. All oral administrations were initiated 7 days after the induction of arthritis and were continued for a total of 12 days. At the end of the experiment, serum aminotransferase, albumin, blood urea nitrogen, creatinine, leukocyte, and inflammatory cytokines [tumor necrosis factor (TNF)-a, interleukin (IL)-1 $\beta_{\text {, }}$ and IL-6] were analyzed. Hematoxylin and eosin (H\&E) and safranin O-fast green staining of the articular structures of the knee joint were performed.

Results: TNF- $\alpha$ and IL-1 $\beta$ decreased in the ChondroT100 and ChondroT200 groups compared with those in the control group. IL-6 and aspartate aminotransferase decreased in the ChondroT50, ChondroT100, and ChondroT200 groups compared with that in the control group. Albumin, WBC and lymphocytes decreased in the ChondroT100 and ChondroT200 groups compared with those in the control group. In H\&E stain, synoviocytes, cartilage lacunae, and chondrocytes were well preserved in the ChondroT100 and ChondroT200 groups, and safranin O-fast staining showed a clear reaction of proteoglycans in the ChondroT100 and ChondroT200 groups.
\end{abstract}

Conclusions: Based on these results, it can be proposed that ChondroT has anti-osteoarthritic effects on collagenase-induced rat model.

Keywords: Osteoarthritis, Collagenase, ChondroT, Inflammatory cytokine

\footnotetext{
* Correspondence: nakugi@hanmail.net; mofoster@hanmail.net

${ }^{2}$ College of Korean Medicine, Dongshin University, 185 Geonjae-ro, Naju-si, Jeollanam-do 58245, Republic of Korea

${ }^{1}$ Department of Rehabilitation Medicine of Korean Medicine, Mokpo Korean

Hospital of Dongshin University, 313 Baengnyeon-daero, Mokpo-si 58665,

Republic of Korea

Full list of author information is available at the end of the article
} International License (http://creativecommons.org/licenses/by/4.0/), which permits unrestricted use, distribution, and reproduction in any medium, provided you give appropriate credit to the original author(s) and the source, provide a link to the Creative Commons license, and indicate if changes were made. The Creative Commons Public Domain Dedication waiver (http://creativecommons.org/publicdomain/zero/1.0/) applies to the data made available in this article, unless otherwise stated. 


\section{Background}

Osteoarthritis (OA), known to be associated with a number of clinical symptoms, has become a significant problem worldwide, due to an increase in the aging population [1]. It results in cartilage degradation which, in turn, leads to cartilage bone damage [2]. This cartilage degradation has been recognized to be induced by inflammatory cytokines, such as IL-6, IL- $1 \beta$, and TNF- $\alpha[3,4]$. Upon mechanical stimulation, the cartilage matrix exhibits changes such that the IL- 1 and TNF- $\alpha$ are destroyed and reformed by cytoplasmic decomposing enzymes $[5,6]$. This process results in the occurrence of joint ankylosis, progressive motor disturbance, pain near joints, tumentia, and redness [7, 8].

Ganghwaljetongyeum (GHJTY) is a traditional Korean herbal medicine commonly used to treat joint pain, limitation of motion, fever, and swelling and to inhibit inflammatory processes associated with arthritis [9]. Our previous study showed that GHJTY which is a complex herbal decoction composed of 18 plants may be effective in attenuating rheumatoid arthritis by inhibiting the production of pro-inflammatory mediators and proliferation of synoviocytes [10].

In order to improve the efficacy and convenience of pharmaceutical prescription, a previous study used bioinformatics to identify the five medicinal herbs with the greatest potential [11]. The five herbs were then combined in a 6:4:4:4:3 ratio, and the water extract solution was named ChondroT, a new complex herbal medication.

ChondroT exhibited a chondroprotective effect and demonstrated multi-target mechanisms related to inflammation and arthritis [12]. In addition, the suppressive effect was greater than that exhibited by GHJTY, suggesting that it had therapeutic potential for the treatment of arthritis [12]. Moreover, it was also found to be effective in treating a rat model of rheumatoid arthritis(RA), where it significantly suppressed the progression of complete Freund's adjuvant-induced arthritis, evident from a decrease in paw and knee joint swelling. It was also effective in preventing articular cartilage and synovial tissue degeneration [13].

Injecting collagenase into the glenoid cavity is a method commonly used to induce arthritis in rats [14]. It damages the ligament and meniscus and decreases stability, forcing the mechanical stress to affect the synovial cell and cartilage cell metabolism, thereby producing symptoms similar to osteoarthritis [15]. For this reason, collagenase induced rat model is typically used to investigate therapeutic agents with anti-osteoarthritic potential [16].

This was done by analyzing the inflammatory cytokines (TNF- $\alpha$, IL-1 $\beta$, and IL-6), performing hematoxylin
\& eosin $(\mathrm{H} \& \mathrm{E})$ staining and safranin $\mathrm{O}$-fast green staining of the articular structures of the knee joint to investigate the effects of ChondroT in collagenase-induced osteoarthritis rat model.

\section{Methods \\ Animals}

Adult male Sprague-Dawley rats weighing 170-180 g were housed in a room with constant temperature $\left(24-26{ }^{\circ} \mathrm{C}\right.$ ) and humidity (40\%-60\%). Food (Pellet, GMO, Korea) and water were available ad libitum. Animals were acclimated to the laboratory environment for 1 week before commencement of the experiment, and all procedures were approved by the Institutional Animal Care and Use Committee of the Dongshin University (DSU-20151003-01). After the experiment, all the rats were euthanized using Carbon dioxide $(\mathrm{CO} 2)$ in accordance with AVMA guidelines.

\section{Collagenase-induced arthritis and oral Administration of Drug} Arthritis was induced by dissolving $1.5 \mathrm{KU}$ of Collagenase (Type VII: Sigma, USA) in $600 \mu \mathrm{L}$ of $0.9 \%$ sterile saline followed by injection into the left hind knee joint. Forty-two rats were randomly divided into seven groups $(n=6)$ as follows: intact, control, three ChondroT (50, 100 , or $200 \mathrm{mg} / \mathrm{kg}$ ), and two positive control groups (indomethacin $2 \mathrm{mg} / \mathrm{kg}$, Joins Tab. $20 \mathrm{mg} / \mathrm{kg}$ ). Oral administration of ChondroT was initiated on the 7 th day after arthritis induction, and was continued for 12 days thereafter. Animals were anesthetized using $2.5 \%$ isoflurane.

\section{Preparation of herbal materials}

The five herbal medicines constituting ChondroT were purchased from Omniherb Co. (Yeongcheon, Korea), and their origin was taxonomically confirmed by Professor Jong-Kil Jeong at the Department of Herbology, College of Oriental Medicine, Dongshin University.

The five herbs (OK, LJ, AG, CM, and PA) were combined in a 6:4:4:4:3 ratio (Table 1 ). ChondroT was prepared by carrying out water extraction once, using 10 -fold solvent at $100{ }^{\circ} \mathrm{C}$ for $3 \mathrm{~h}$, and then filtering it (180 mesh). The water extract solution was concentrated using a continuous vacuum evaporator (approximately $55-60{ }^{\circ} \mathrm{C}, 670 \mathrm{mmHg}$ ), and this was followed by vacuum drying using a vacuum drier $(720 \mathrm{mmHg})$ for 8 h. The yield was approximately $29.4 \%$. Voucher specimens (No. GHJTY 501) of the collected herb samples were deposited in the herbarium of Jung Woo Shin Yak (Asan, Korea). 
Table 1 Composition of ChondroT

\begin{tabular}{|c|c|c|c|c|}
\hline Latin name & Scientific name & Family & Rate & Source \\
\hline Osterici Radix & Ostericum koreanum Maximowicz & Umbelliferae & 6 & Korea \\
\hline Angelicae Gigantis Radix & Angelica gigas Nakai & Umbelliferae & 4 & Korea \\
\hline Clematidis Radix & Clematis manshurica Ruprecht & Ranunculaceae & 4 & China \\
\hline Lonicerae Folium & Lonicera japonica Thunberg & Caprifoliaceae & 4 & China \\
\hline Phellodendri Cortex & Phellodendrom amurense Ruprecht & Rutaceae & 3 & China \\
\hline
\end{tabular}

\section{Reagents and high-performance liquid chromatography (HPLC) analysis}

Chlorogenic acid (1) and berberine chloride (2) were purchased from U.S. Pharmacopeial Convention (Rockville, MD, USA), and Decursin (3) was purchased from ChemFaces Biochemical Co. Ltd. (Wuhan, China). The purities of the reference compounds 1-3 were $97.3 \%, 81.0 \%$, and $99.4 \%$, respectively, measured using HPLC analysis. The chemical structures of these compounds have been shown in Fig. 1a. HPLC-grade methanol, acetonitrile and water were obtained from J.T. Baker (Phillipsburg, NJ, USA), while analytical grade formic acid was purchased from Sigma-Aldrich (St. Louis, MO, USA).

For quality control of the ChondroT sample, all experiments were performed using a Shimadzu Prominence LC-20A Series (Shimadzu, Kyoto, Japan) equipped with a solvent delivery unit (LC-20AT), online degasser (DGU-20A3R), column oven (CTO-20 AC), autosampler (SIL-20 AC), and UV-VIS detector (SPD20A). Data acquisition and processing were conducted using Lab solution software (version 5.73 SP3, Kyoto,
Japan). Compounds 1-3 were separated using a Waters SunFire C18 column $(4.6 \times 250 \mathrm{~mm} ; 5 \mu \mathrm{m}$, Milford, MA, USA) maintained at $40{ }^{\circ} \mathrm{C}$. The mobile phases consisted of (A) $0.1 \%(\mathrm{v} / \mathrm{v})$ aqueous formic acid and (B) $0.1 \%(\mathrm{v} / \mathrm{v})$ formic acid in acetonitrile, and the gradation conditions were optimized as follows: a duration range of $0-10 \mathrm{~min}, 10 \%-35 \% \mathrm{~B} ; 10-30 \mathrm{~min}, 35 \%-$ 65\% B; 30-35 $\mathrm{min}, 65 \%-80 \% \mathrm{~B} ; 35-40 \mathrm{~min}, 80 \% \mathrm{~B}$; $40-41 \mathrm{~min}, 80 \%-10 \% \mathrm{~B}$; and $41-50 \mathrm{~min}, 10 \% \mathrm{~B}$. The flow rate and injection volume were $1.0 \mathrm{~mL} / \mathrm{min}$ and $10 \mu \mathrm{L}$, respectively. For HPLC analysis, lyophilized ChondroT (1 g) was dissolved in $100 \mathrm{~mL}$ of $70 \%$ methanol and extracted for $60 \mathrm{~min}$ by sonication. The extracted solution was centrifuged at $3000 \mathrm{rpm}$ for $10 \mathrm{~min}$, and then passed through a $0.45-\mu \mathrm{m}$ syringe filter before HPLC analysis.

\section{Blood and serum tests}

Blood samples were collected, and $100 \mu \mathrm{L}$ of it was used to measure leukocytes using a Multispecies Hematology Analyzer (950, Hemavet, USA). The rest of the blood sample was used to measure aspartate aminotransferase

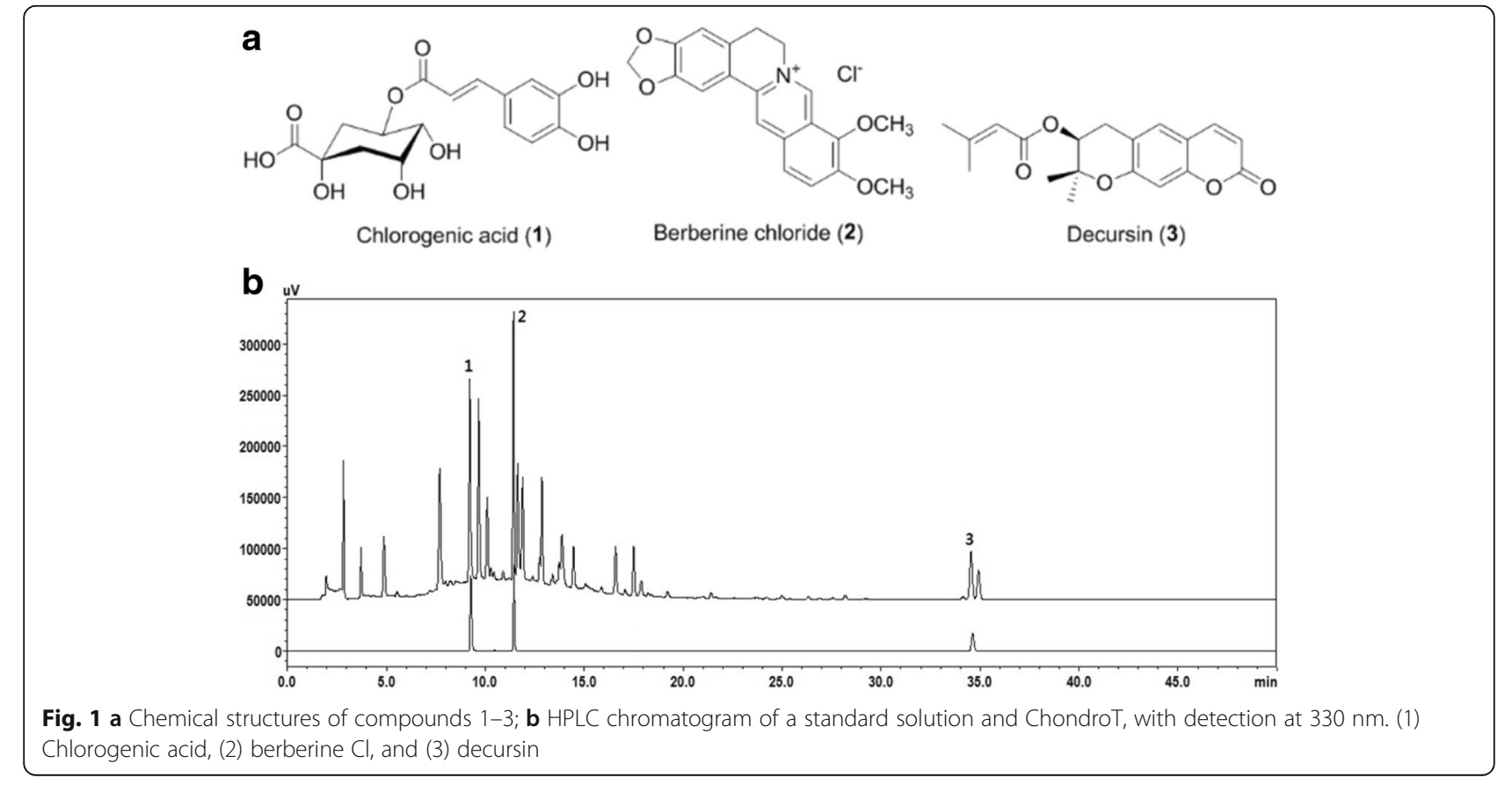


Table 2 Concentrations of the three marker components in the ChondroT by HPLC $(n=3)$

\begin{tabular}{lllll}
\hline Compound & Mean $(\mathrm{mg} / \mathrm{g})$ & $\mathrm{SD}^{\mathrm{a}}$ & $\mathrm{RSD}^{\mathrm{b}}(\%)$ & Source \\
\hline $\begin{array}{l}\text { Chlorogenic } \\
\text { acid }\end{array}$ & 3.67 & 0.08 & 2.20 & Lonicera japonica \\
Berberine Cl & 2.41 & 0.22 & 8.99 & Phellodendron amurense \\
Decursin & 1.87 & 0.18 & 9.57 & Angelica gigas \\
\hline
\end{tabular}

${ }^{\mathrm{a}}$ standard deviation, ${ }^{\mathrm{b}}$ relative standard deviation

(AST), alanine transaminase (ALT), albumin, BUN, and creatinine levels with the help of a high-speed centrifuge (VS-6000CFi, Korea) at $3000 \mathrm{rpm}$ for $20 \mathrm{~min}$.

\section{Measurement of TNF- $\alpha$, IL- $1 \beta$, and IL- 6}

TNF- $\alpha$ was measured using a Rat TNF- $\alpha$ kit (Invitrogen, USA), IL-1 $\beta$ was assessed using a Rat IL-1 $\beta$ kit (R\&D Systems, USA) and IL-6 was evaluated using a Rat IL-6 kit (Invitrogen, USA). The optical densities (OD) of all samples were measured at $450 \mathrm{~nm}$ using Spectramax (M2, Molecular Devices, USA).

\section{Hematoxylin and eosin staining}

The left knee joint was removed and fixed in Bouin solution for $>24 \mathrm{~h}$. Decalcification was performed using a $2.5 \%$ nitric acid solution, which was changed once a day for 7 days. The removed tissue was dehydrated using a Tissue Processor (Tissue-Tek II, Japan). After deparaffinization and staining with H\&E (Muto, Japan), the samples were observed under an optical microscope (Nikon, Japan).

\section{Safranin O-fast staining}

After deparaffinization, the left knee joint was treated with Weigert's Iron Hematoxylin solution (Sigma, USA) for $10 \mathrm{~min}$ and stained with $0.001 \%$ Fast Green solution (Sigma, USA) for $5 \mathrm{~min}$. The sample was then incubated with $1 \%$ acetate solution for $10 \mathrm{~s}$ and stained with $0.1 \%$

Table 3 Effect of ChondroT on TNF- $a$, IL-1 $\beta$, and IL-6 levels in rats with collagenase -induced arthritis

\begin{tabular}{llll}
\hline Groups & TNF- $\mathrm{a}(\mathrm{pg} / \mathrm{mL})$ & $\mathbb{I L}-1 \beta(\mathrm{pg} / \mathrm{mL})$ & $\mathbb{I L}-6(\mathrm{pg} / \mathrm{mL})$ \\
\hline Intact & $2.67 \pm 0.37^{\mathrm{b}}$ & $11.69 \pm 0.42^{\mathrm{c}}$ & $23.47 \pm 2.28^{\mathrm{c}}$ \\
Control & $5.48 \pm 0.43^{\mathrm{a}}$ & $16.06 \pm 0.59^{\mathrm{a}}$ & $70.45 \pm 8.52^{\mathrm{a}}$ \\
Indomethacin & $3.14 \pm 0.24^{\mathrm{b}}$ & $13.24 \pm 0.70^{\mathrm{bc}}$ & $40.67 \pm 1.88^{\mathrm{b}}$ \\
Joins tab & $2.19 \pm 0.34^{\mathrm{b}}$ & $12.66 \pm 0.73^{\mathrm{bc}}$ & $31.74 \pm 1.78^{\mathrm{bc}}$ \\
ChondroT50 & $3.82 \pm 0.68^{\mathrm{b}}$ & $14.17 \pm 0.62^{\mathrm{b}}$ & $44.19 \pm 4.03^{\mathrm{b}}$ \\
ChondroT100 & $2.89 \pm 0.70^{\mathrm{b}}$ & $13.41 \pm 0.35^{\mathrm{bc}}$ & $34.58 \pm 1.73^{\mathrm{bc}}$ \\
ChondroT200 & $2.77 \pm 0.46^{\mathrm{b}}$ & $13.07 \pm 0.50^{\mathrm{bc}}$ & $34.40 \pm 2.03^{\mathrm{bc}}$ \\
F Value & 4.603 & 4.87 & 10.56 \\
P Value & 0.0017 & 0.0012 & 0.0001 \\
\hline
\end{tabular}

All values are mean \pm standard error. Values with different superscripts within the same row are significantly different at $p<0.05$ by LSD multiple range test safranin O solution (Sigma, USA) for $5 \mathrm{~min}$. Thereafter, the tissue was dehydrated and observed under an optical microscope (Nikon, Japan).

\section{Statistical analysis}

All statistical analyses were performed in SAS 9.1 version for Windows. A one-way analysis of variance was performed on each group, and the results have been expressed as mean \pm standard error (SE). Comparisons between groups were performed using a post hoc least
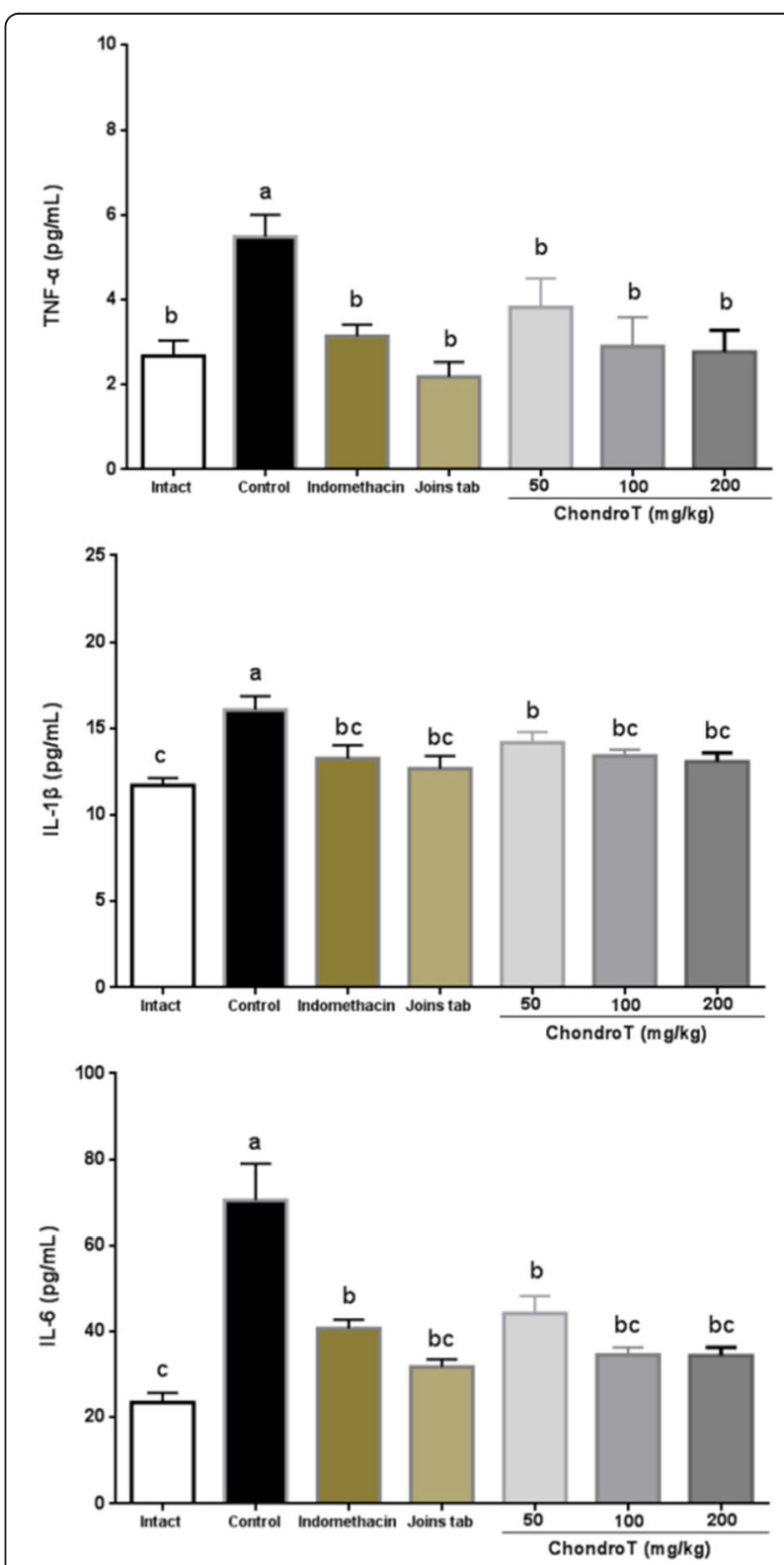

Fig. 2 Effect of ChondroT on TNF-a, IL-1 $\beta$, and IL-6 levels in rats with collagenase -induced arthritis 
significant difference test. $p$ values of $<0.05$ and $<0.01$ were considered statistically significant.

\section{Results}

Quality assessment of three marker components in ChondroT

Quality assessment of ChondroT was performed using three marker compounds and HPLC. The selected compounds were as follows: compound 1 (Lonicerae Folium), compound 2 (Phellodendri Cortex), and compound 3 (Angelicae Gigantis Radix). All analytes were separated within $40 \mathrm{~min}$, and the typical chromatogram of a $70 \%$ methanol extract of ChondroT has been shown in Fig. 1b. Quantification was performed by UV-VIS detection at $330 \mathrm{~nm}$ based on retention time. The retention times of components $1-3$ were $9.25,11.35$, and $34.51 \mathrm{~min}$, respectively. Under optimized chromatography conditions, the concentrations of the marker compounds $1-3$ in ChondroT were $3.67 \pm 0.08,2.41 \pm 0.22$, and $1.87 \pm 0.18 \mathrm{mg} / \mathrm{g}$, respectively (Table 2, Fig. 1).

\section{Effect of ChondroT on Proinflammatory cytokines}

With regard to the effect of the amount of ChondroT administered on anti-inflammatory and inflammationinducing agents in serum, a significant increase in the level of TNF- $\alpha$ was observed as compared to the intact group. The indomethacin, Joins tab, ChondroT100, and ChondroT200 groups also showed a significant increase compared with the control group.

With regard to the level of IL- $1 \beta$, the control group showed a significant increase as compared to the intact group, while the indomethacin, Joins tab, ChondroT100, and ChondroT200 groups showed a significant decrease as compared to the control group. In the case of the level of IL-6, the control group showed a significant increase as compared to the intact group, while the indomethacin, Joins tab, ChondroT50, ChondroT100, and ChondroT200 groups showed a significant decrease as compared to the control group. and in ChondroT100 and ChondroT200 groups, TNF- $\alpha$, IL- $1 \beta$, and IL- 6 were equivalent as compared with those in the indomethacin and Joins tab (Table 3, Fig. 2).

\section{Effect of ChondroT on histopathological changes as assessed by hematoxylin and eosin staining} Histopathologically, a small portion of synovial membrane was seen to be destroyed in the control group, while the indomethacin and Joins tab groups clearly exhibited synovial membrane cells of cartilage and synovial cavity compared to the control group. In addition, the ChondroT100 and ChondroT200 groups exhibited synovial membrane cells, synovial cavity, and cartilage cells similar to the intact group (Fig. 3).

\section{Effect of ChondroT on histopathological changes assessed with safranin O-fast staining}

Safranin O-fast staining showed that the positive reaction of proteoglycans in articular cartilage in the control group was lower than that of the intact group. Also, in the ChondroT200 group, the deep layer of cartilage showed a higher positive reaction for proteoglycans as compared to the control group (Fig. 4).

\section{Leukocyte}

With regard to the effect of ChondroT administration on leukocyte, eosinophils in the control group exhibited a significant decrease compared with those in the intact group, whereas WBC, neutrophils, lymphocytes, and monocytes exhibited no significant differences. WBC and lymphocytes exhibited a significant decrease in ChondroT100 and ChondroT200 groups compared with those in the control group (Table 4, Fig. 5).
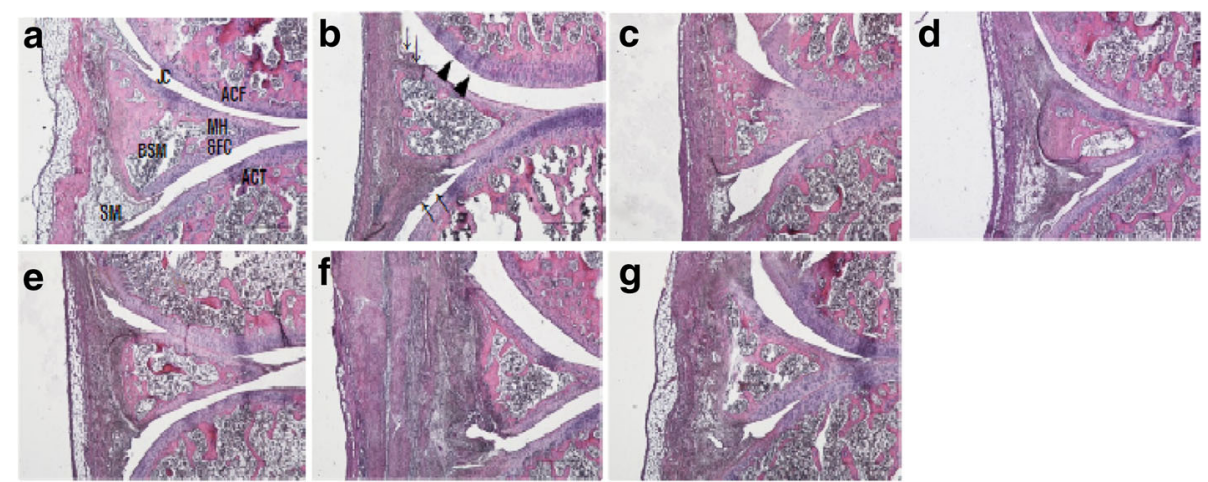

Fig. 3 Histopathological changes (H\&E stain) in the knee joint after ChondroT administration in collagenase induced arthritis rats. In H\&E stain, synoviocytes, cartilage lacunae, and chondrocytes were well preserved in the ChondroT100 and ChondroT200 groups. HE-stain, Scale bars = $500 \mu \mathrm{m}$. a Intact, b Control, c Indomethacin, d Joins Tab, e ChondroT50, f ChondroT100, g ChondroT200 


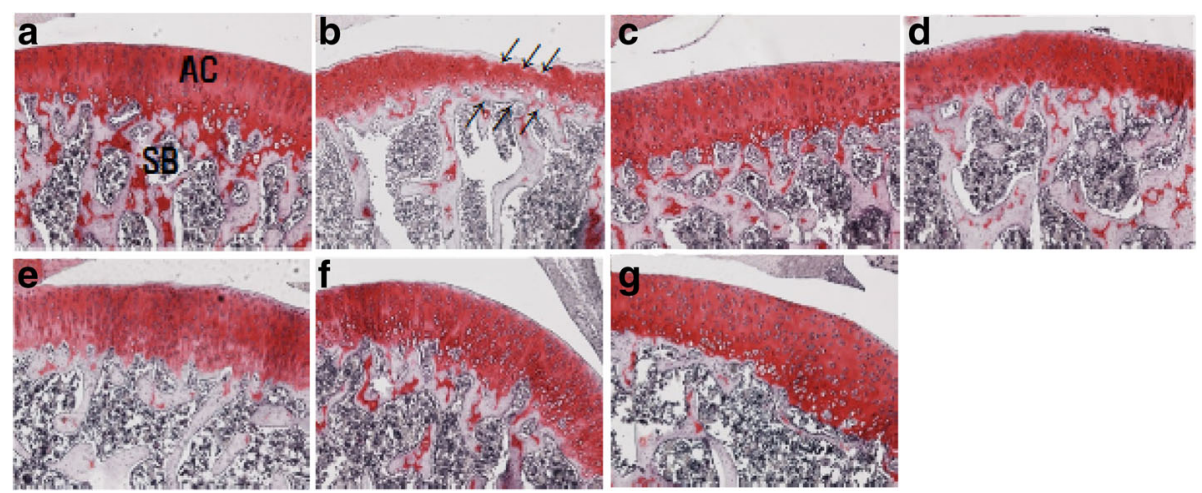

Fig. 4 Histopathological changes (Safranin O-fast staining) in the knee joint after ChondroT administration in collagenase-induced arthritis rats. Safranin O-fast staining showed a clear reaction of proteoglycans in ChondroT200 group. Safranin O-fast stain, Scale bars $=100 \mu$ m. a Intact, $\mathbf{b}$ Control, c Indomethacin, d Joins Tab, e ChondroT50, f ChondroT100, g ChondroT200

\section{Effect of ChondroT on aminotransferase}

The collagenase induced control group exhibited a significant increase in AST compared to the intact group, while the indomethacin, Joins tab, ChondroT50, ChondroT100, and ChondroT200 groups showed a significant decrease compared with the control group. In the case of ALT, a decreasing tendency was observed, although this was not statistically significant (Table 5, Fig. 6).

\section{Effect of ChondroT on albumin, BUN, and creatinine}

The ChondroT100 and ChondroT200 groups showed a significant decrease in albumin as compared to the control group while there were no significant differences with regard to BUN and creatinine (Table 6, Fig. 7).

\section{Discussion}

The treatment of osteoarthritis can be divided into nonpharmacologic treatment, drug treatment and surgical treatment [17]. Acetaminophen is commonly used as the primary drug along with non-steroidal anti-inflammatory drugs (NSAIDs), such as naproxen, aceclofenac, and celecoxib [18]. Besides, narcotic analgesics, such as oxymorphone, oxycodone, and oxytrex, and parenteral injections of hyaluronic acid in the joint are used selectively based on the medical team's diagnosis. Surgical treatment is selectively used for patients with severe pain and impaired functioning [19-21].

NSAIDs are the drugs most frequently used for the treatment of pain from osteoarthritis, and are 30\% more effective in eliminating pain than high dose acetaminophen $[21,22]$. However, it does not have any effect on some patients, and has several side effects on the upper gastrointestinal tract including indigestion, vomiting and ulcer edema [23]. Additionally, since the action that particularly suppresses cyclooxygenase-2, also has a side effect on the cardiovascular system, its use is limited [24]. Thus, a safer and more effective medicine is required [21, 22].

Therefore, oriental medical treatment has been actively studied, as it is effective against osteoarthritis and has fewer side effects. However, most of these studies use existing prescriptions, and there are very few newly developed oriental medicines. This study verified the

Table 4 Changes in the blood WBC, neutrophils, lymphocytes, monocytes, and eosinophils contents after ChondroT administration in collagenase-induced arthritis rats

\begin{tabular}{llllll}
\hline Groups & WBC $(K / \mu L)$ & $N E(K / \mu L)$ & $L Y(K / \mu L)$ & $M O(K / \mu L)$ & $E O(K / \mu L)$ \\
\hline Intact & $4.10 \pm 0.84^{\mathrm{ab}}$ & $0.56 \pm 0.06^{\mathrm{a}}$ & $3.38 \pm 0.80^{\mathrm{abc}}$ & $0.14 \pm 0.03^{\mathrm{ab}}$ & $0.02 \pm 0.01^{\mathrm{a}}$ \\
Control & $5.47 \pm 0.57^{\mathrm{a}}$ & $0.68 \pm 0.11^{\mathrm{a}}$ & $4.60 \pm 0.48^{\mathrm{a}}$ & $0.18 \pm 0.02^{\mathrm{ab}}$ & $0.01 \pm 0.00^{\mathrm{a}}$ \\
Indomethacin & $4.49 \pm 0.40 \mathrm{c}$ & $0.61 \pm 0.06^{\mathrm{a}}$ & $3.68 \pm 0.34^{\mathrm{abc}}$ & $0.19 \pm 0.03^{\mathrm{ab}}$ & $0.01 \pm 0.00^{\mathrm{a}}$ \\
Joins tab & $4.59 \pm 0.39^{\mathrm{ab}}$ & $0.66 \pm 0.17^{\mathrm{a}}$ & $3.67 \pm 0.24^{\mathrm{abc}}$ & $0.18 \pm 0.02^{\mathrm{ab}}$ & $0.03 \pm 0.03^{\mathrm{a}}$ \\
ChondroT50 & $5.48 \pm 0.76^{\mathrm{a}}$ & $0.75 \pm 0.15^{\mathrm{a}}$ & $4.51 \pm 0.62^{\mathrm{ab}}$ & $0.20 \pm 0.02^{\mathrm{a}}$ & $0.02 \pm 0.01^{\mathrm{a}}$ \\
ChondroT100 & $3.79 \pm 0.17^{\mathrm{b}}$ & $0.45 \pm 0.04^{\mathrm{a}}$ & $3.21 \pm 0.18^{\mathrm{bc}}$ & $0.12 \pm 0.03^{\mathrm{b}}$ & $0.01 \pm 0.00^{\mathrm{a}}$ \\
ChondroT200 & $3.70 \pm 0.34^{\mathrm{b}}$ & $0.53 \pm 0.03^{\mathrm{a}}$ & $3.04 \pm 0.37^{\mathrm{c}}$ & $0.13 \pm 0.02^{\mathrm{b}}$ & $0.01 \pm 0.00^{\mathrm{a}}$ \\
F Value & 1.81 & 0.95 & 1.65 & 1.79 & 0.79 \\
$P$ Value & 0.1342 & 0.4752 & 0.1706 & 0.1368 & 0.5878 \\
\hline
\end{tabular}

All values are mean \pm standard error. Values with different superscripts within the same row are significantly different at $p<0.05$ by LSD multiple range test 

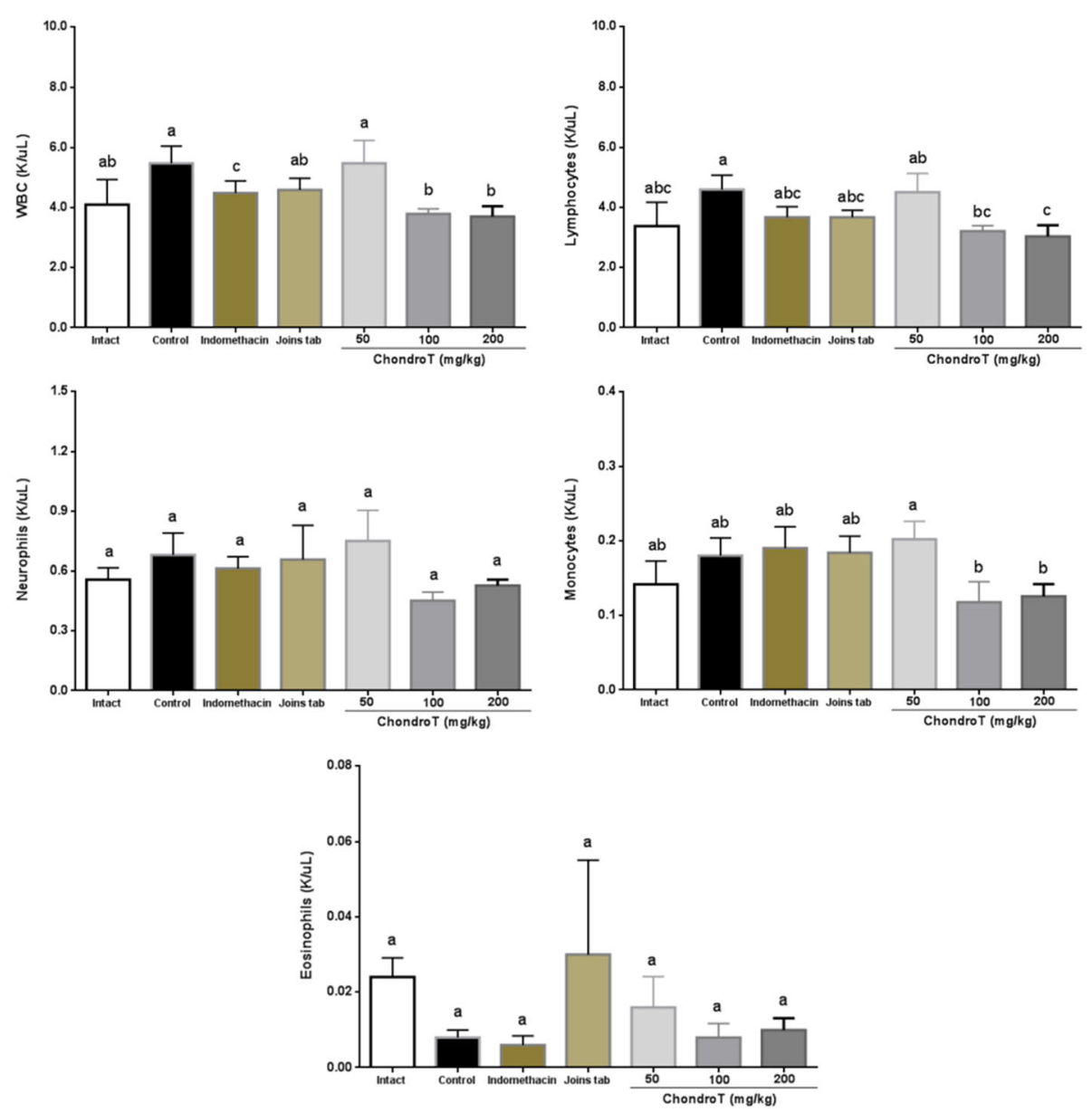

Fig. 5 Changes in the blood WBC, neutrophils, lymphocytes, monocytes, and eosinophils contents after ChondroT administration in collagenase-induced arthritis rats

effects of ChondroT, a newly developed oriental medicine, on osteoarthritis using gene data processing techniques.

The indomethacin used in the positive control group is an NSAID used to treat osteoarthritis, inflammatory periodontal disease, and ankylosing spondylitis [25-28].

Table 5 Changes in serum aminotransferase content after ChondroT administration in collagenase-induced arthritis rats

\begin{tabular}{lll}
\hline Groups & AST $(U / L)$ & ALT $(U / L)$ \\
\hline Intact & $88.3 \pm 8.36^{\mathrm{ab}}$ & $36.3 \pm 2.91^{\mathrm{a}}$ \\
Control & $101.2 \pm 3.94^{\mathrm{a}}$ & $43.8 \pm 3.17^{\mathrm{a}}$ \\
Indomethacin & $84.8 \pm 3.31^{\mathrm{bc}}$ & $38.2 \pm 3.10^{\mathrm{a}}$ \\
Joins tab & $72.8 \pm 1.13^{\mathrm{c}}$ & $36.0 \pm 2.52^{\mathrm{a}}$ \\
ChondroT50 & $76.4 \pm 0.62^{\mathrm{bc}}$ & $36.0 \pm 1.35^{\mathrm{a}}$ \\
ChondroT100 & $83.3 \pm 4.20^{\mathrm{bc}}$ & $37.3 \pm 2.80^{\mathrm{a}}$ \\
ChondroT200 & $81.5 \pm 4.77^{\mathrm{bc}}$ & $35.0 \pm 2.56^{\mathrm{a}}$ \\
F Value & 3.204 & 0.9025 \\
$P$ value & 0.0155 & 0.5066 \\
\hline
\end{tabular}

All values are mean \pm standard error. Values with different superscripts within the same row are significantly different at $p<0.05$ by LSD multiple range test
Joins tab is a natural drug comprising Korean virgin's bower, Trichosanthes kirilowii Maxim, and Thesium and is used to treat degenerative arthritis and rheumatoid arthritis [29]. It is a newly developed drug that suppresses the destruction of cartilage and activation of joint catabolic enzymes, in addition to its simple antiinflammatory and analgesic effects [29-31].

TNF- $\alpha$ is a high-level cytokine that induces catabolism, allowing generation of proteoclastic enzymes that destroy the cartilage, produced by activated monocyte and macrophagocyte, stimulating the osteoclasts gathering in the region of the topical bone resorption, leading inflammatory reaction, contributing to removing topical minerals [32]. IL- $1 \beta$ is known as a powerful cytokine that induces the dissolution of cartilage, produces inflammatory agents, such as prostaglandin E2 and nitric oxide, from the cartilage and synovial cells, and stimulates expression of matrix metalloproteinases [33]. It has been reported that IL-6 exhibits higher activity in the joint fluid than in the serum, suggesting an important role in osteoarthritis. It also facilitates the proliferation of synovial cells and increases the activity of 


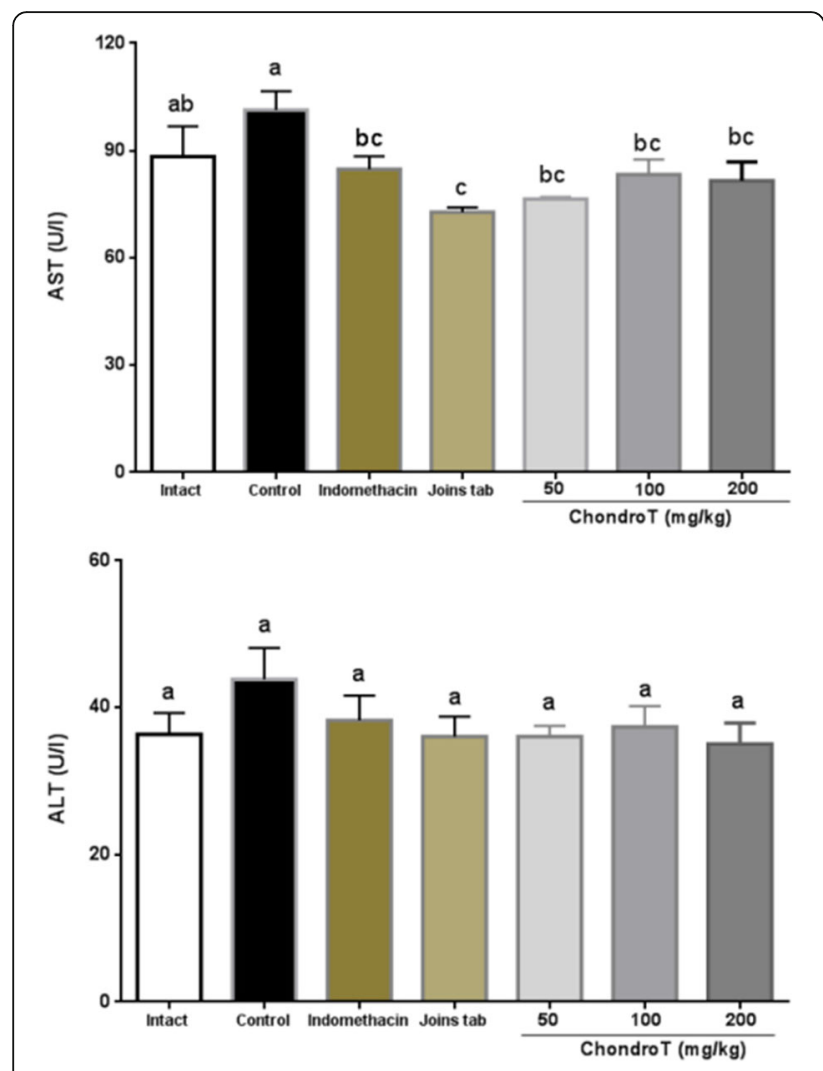

Fig. 6 Changes in serum aminotransferase content after ChondroT administration in collagenase-induced arthritis rats

osteoclasts, forming the pannus and producing proteoclastic enzymes that destroy the cartilage joint $[34,35]$.

As a result, in the effects of the volume of ChondroT on a change in TNF- $\alpha$ and IL-1 $\beta$, there was no significant decrease in ChondroT50 group as compared to the control group. While there were significant decreases in ChondroT100 and ChondroT200 group, and there were significant decreases in a change in IL-6 in all ChondroT50,

Table 6 Changes in serum albumin, BUN, and creatinine content after ChondroT administration in collagenase-induced arthritis rats

\begin{tabular}{llll}
\hline Groups & Albumin $(\mathrm{g} / \mathrm{dL})$ & BUN $(\mathrm{mg} / \mathrm{dL})$ & Creatinine $(\mathrm{mg} / \mathrm{dL})$ \\
\hline Intact & $3.5 \pm 0.11^{\mathrm{ab}}$ & $11.8 \pm 0.59^{\mathrm{b}}$ & $0.17 \pm 0.02^{\mathrm{a}}$ \\
Control & $3.8 \pm 0.07^{\mathrm{a}}$ & $13.5 \pm 0.80^{\mathrm{ab}}$ & $0.18 \pm 0.01^{\mathrm{a}}$ \\
Indomethacin & $3.7 \pm 0.10^{\mathrm{a}}$ & $12.9 \pm 1.29^{\mathrm{ab}}$ & $0.14 \pm 0.02^{\mathrm{a}}$ \\
Joins tab & $3.5 \pm 0.07^{\mathrm{ab}}$ & $14.9 \pm 0.46^{\mathrm{a}}$ & $0.13 \pm 0.02^{\mathrm{a}}$ \\
ChondroT50 & $3.6 \pm 0.05^{\mathrm{a}}$ & $14.5 \pm 0.56^{\mathrm{a}}$ & $0.20 \pm 0.03^{\mathrm{a}}$ \\
ChondroT100 & $3.4 \pm 0.10^{\mathrm{b}}$ & $14.5 \pm 0.37^{\mathrm{a}}$ & $0.15 \pm 0.02^{\mathrm{a}}$ \\
ChondroT200 & $3.4 \pm 0.02^{\mathrm{b}}$ & $12.9 \pm 1.26^{\mathrm{ab}}$ & $0.15 \pm 0.03^{\mathrm{a}}$ \\
F Value & 2.63 & 1.84 & 0.35 \\
P Value & 0.0370 & 0.1250 & 0.9054 \\
\hline
\end{tabular}

All values are mean \pm standard error. Values with different superscripts within the same row are significantly different at $p<0.05$ by LSD multiple range test
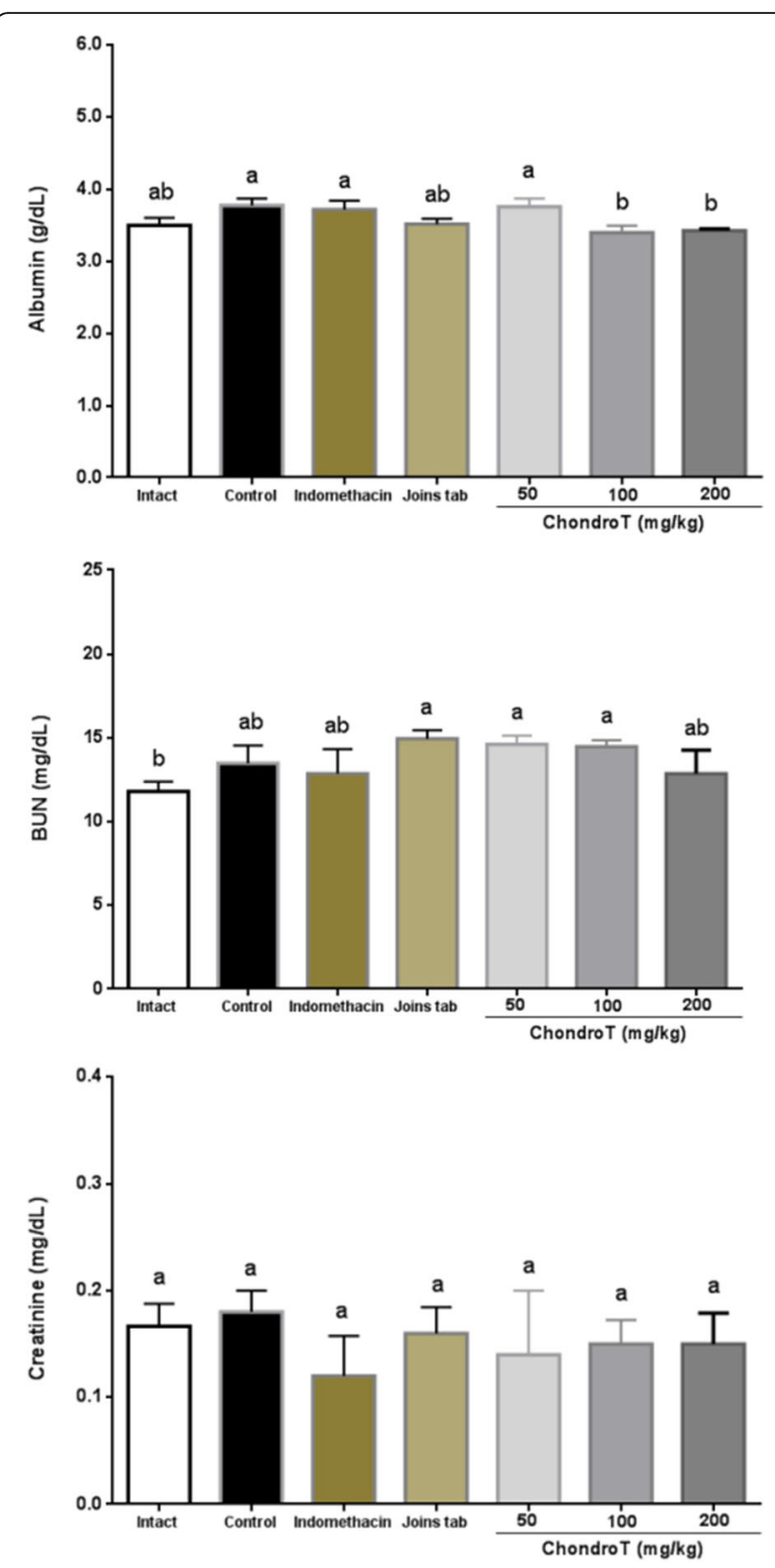

Fig. 7 Changes in serum albumin, BUN, and creatinine content after ChondroT administration in collagenase-induced arthritis rats

ChondroT100 and ChondroT200 groups as compared to the control group. so it is assumed that ChondroT has an effect on the suppression of the inflammatory reaction of osteoarthritis.

$\mathrm{H} \& \mathrm{E}$ and safranin O-fast staining are used for histopathological observation of synovial tissues, cartilage cells, and fibrous tissues in the knee joint. H\&E staining of nucleus, proteoglycan, and protoplasm allows observation of changes in the ingredients easily as Hematoxylin is basophilic while eosin is acidophilic [36]. In safranin O-fast staining, a cationoid chromophyll is combined with an anion like keratan sulfate or 
chondroitin sulfate, which stains ingredients red or orange in proportion to the volume of proteoglycan distributed in the cartilage so as to allow estimation of any changes in the concentration $[37,38]$.

As a result of an observation after H\&E staining, as compared to the control group, in ChondroT100 and ChondroT200 group, there were clear reactions of synovial cells, lacunae and cartilage cells as in the intact group, and as a result of an observation with safranin Ofast staining, there were no big differences in ChondroT50 and ChondroT100 group as compared to the control group while in ChondroT200 group, there was a higher observation of a positive reaction of proteglycans in the cartilage layer of the internal layer adjacent to the bone tissue as compared to the control group. Through the above experiment, it is noted that ChondroT200 has a positive impact on the pathological condition of the arthritis tissue.

Based on the findings of this study, it can be concluded that ChondroT suppresses inflammatory cytokines, reduces the activation of an immune response, inhibits loss of articular cartilage and protects the articular surface. Therefore, we confirmed that chondroT administration was effective for inhibiting the progression of arthritis and for protecting joints.

\section{Conclusions}

ChondroT, a new complex herbal medication, was effective in treating a rat model of arthritis. Specifically, ChondroT significantly suppressed inflammation by a decrease in the proinflammatory cytokines, TNF- $\alpha$, IL$1 \beta$, and IL-6. and it was effective in preventing articular cartilage and synovial tissue degeneration in the histophologic finding. It suggests that ChondroT, a new complex herbal medication, has therapeutic potential for the treatment of arthritis.

\section{Abbreviations}

IL-1 $\beta$ : Interleukin-1 $\beta$; ALT: Alanine transaminase; AST: Aspartate aminotransferase; BUN: Blood urea nitrogen; CFA: Complete Freund's adjuvant; GHJTY: Ganghwaljetongyeum; H\&E: Hematoxylin and eosin; HPLC: High-performance liquid chromatography; NSAIDs: Non-steroidal antiinflammatory drugs; OA: Osteoarthritis; OD: Optical densities; RSD: Relative standard deviation; SD: Standard derivation; SE: Standard error; TNF-a: Tumor necrosis factor-a

\section{Acknowledgments}

This research was supported by a grant of the Korea Health Technology R\&D Project through the Korea Health Industry Development Institute (KHIDI), funded by the Ministry of Health \& Welfare, Republic of Korea (grant number: HI17C0911)

\section{Funding}

This research was supported by a grant of the Korea Health Technology R\&D Project through the Korea.

Health Industry Development Institute (KHIDI), funded by the Ministry of Health \& Welfare, Republic of Korea (grant number: HI17C0911).

\section{Availability of data and materials}

The datasets used and/or analysed during the current study are available from the corresponding author on reasonable request.

\section{Authors' contributions}

JJW conducted data collection, analysis, and preparation of manuscript; GDW and MYJ made substantial contributions to conception, design, and virological experimentation; BGJ and KSG was involved in drafting the manuscript and critically revising it for important intellectual content; $\mathrm{CCH}$, and KYR were involved in critically revising the manuscript for important intellectual content and have approved the final version of the manuscript to be published; NCS and KSJ supervised the study, were the project leader, and helped in drafting the manuscript. All authors have read and approved the final manuscript.

\section{Ethics approval}

All animal experiments were conducted in accordance with the Institutional Animal Care and Use Committee (IACUC) guidelines. All the animal procedures were carried out in accordance with the guidelines of the Animal Care and Use Committee of Dongshin University. The animal study was submitted to, and approved by Animal Care and Use Committee of Dongshin University (Permission number: DSU-2015-1003-01).

\section{Consent for publication}

Not applicable.

\section{Competing interests}

The authors declare that they have no competing interests.

\section{Publisher's Note}

Springer Nature remains neutral with regard to jurisdictional claims in published maps and institutional affiliations.

\section{Author details}

'Department of Rehabilitation Medicine of Korean Medicine, Mokpo Korean Hospital of Dongshin University, 313 Baengnyeon-daero, Mokpo-si 58665, Republic of Korea. ${ }^{2}$ College of Korean Medicine, Dongshin University, 185 Geonjae-ro, Naju-si, Jeollanam-do 58245, Republic of Korea. ${ }^{3}$ Training Department of Bucheon Jaseng hospital of korean medicine, 17 Buil-ro, 191 beon-gil, Bucheon-si, Gyeonggi-do 145598, Republic of Korea. ${ }^{4}$ College of Pharmacy and Research Institute of Drug Development, Chonnam National University, 77 Yongbong-ro, Buk-gu, Gwangju-si 61186, Republic of Korea.

Received: 3 September 2017 Accepted: 28 February 2018 Published online: 19 April 2018

\section{References}

1. Cross M, Smith E, Hoy D, Nolte S, Ackerman I, Fransen M, et al. The global burden of hip and knee osteoarthritis: estimates from the global burden of disease 2010 study. Ann Rheum Dis. 2014;73:1323-30.

2. Kim JH, Ryu KH, Jung KW, Han CK, Kwak WJ, Cho YB. Effects of SKI306X on arachidonate metabolism and other inflammatory mediators. Biol Pharm Bull. 2005;28:1615-20.

3. Vervoordeldonk MJ, Tak PP. Cytokines in rheumatoid arthritis. Curr Rheumatol Rep. 2002:4:208-17.

4. Nishimoto N. Interleukin-6 in rheumatoid arthritis. Curr Opin Rheumatol. 2006;18:277-81.

5. Zeng L, Wang W, Rong XF, Zhong Y, Jia P, Zhou GQ. Chondroprotective effects and multi-target mechanisms of icariin in IL-1 beta-induced human SW 1353 chondrosarcoma cells and a rat osteoarthritis model. Int Immunopharmacol. 2014;18:175-81.

6. Kobayashi M, Squires GR, Mousa A, Tanzer M, Zukor DJ, Antoniou J, et al. Role of interleukin-1 and tumor necrosis factor? In matrix degradation of human osteoarthritic cartilage. Arthritis Rheum. 2005;52:128-35.

7. Chadjichristos C, Ghayor C, Kypriotou M, Martin G, Renard E, Ala-Kokko L, et al. Sp1 and Sp3 transcription factors mediate interleukin-1 $\beta$ downregulation of human type $\|$ collagen gene expression in articular chondrocytes. J Biol Chem. 2003:278:39762-72.

8. Choi HM, Oh DH, Bang JS, Yang H-I, Yoo MC, Kim KS. Differential effect of $\mathrm{IL}-1 \beta$ and TNF- $\alpha$ on the production of $\mathrm{IL}-6, \mathrm{IL}-8$ and PGE2 in fibroblast-like synoviocytes and THP-1 macrophages. Rheumatol Int. 2010;30:1025-33. 
9. Kim YH, Lee JH. CheongKangEuiGam. Seoul: Seongbosa; 2001. p. 315.

10. Jeoung BR, Lee KD, Na CS, Kim YE, Kim B, Kim YR. Ganghwaljetongyeum, an anti-arthritic remedy, attenuates synoviocyte proliferation and reduces the production of proinflammatory mediators in macrophages: the therapeutic effect of GHJTY on rheumatoid arthritis. BMC Complement Altern Med. 2013;13:47-52.

11. Choi W, Choi CH, Kim YR, Kim SJ, Na CS, Lee H. HerDing: herb recommendation system to treat diseases using genes and chemicals. Database. 2016;2016 https://doi.org/10.1093/database/baw011.

12. Park JU, Kim SJ, Na CS, Choi C, Seo CS, Son JK, et al. Chondroprotective and anti-inflammatory effects of ChondroT, a new complex herbal medication. BMC Complement Altern Med. 2016;16:213-23.

13. Kim W, Park S, Choi C, Kim YR, Park I, Seo C, et al. Evaluation of antiinflammatory potential of the new Ganghwaljetongyeum on adjuvantinduced inflammatory arthritis in rats. Evid Based Complement Alternat Med. 2016;2016 https://doi.org/10.1155/2016/1230294.

14. Blom $A B$, van Lent $P L$, Libregts S, Holthuysen $A E$, van der Kraan PM, van Rooijen $\mathrm{N}$, et al. Crucial role of macrophages in matrix metalloproteinasemediated cartilage destruction during experimental osteoarthritis: involvement of matrix metalloproteinase 3. Arthritis Rheum. 2007;56:147-57.

15. Botter SM, van Osch GJVM, Waarsing JH, van der Linden JC, Verhaar JAN, Pols HAP, et al. Cartilage damage pattern in relation to subchondral plate thickness in a collagenase-induced model of osteoarthritis. Osteoarthr Cartil. 2008;16:506-14.

16. Seo BK, Sung WS, Park YC, Baek YH. The electroacupuncture-induced analgesic effect mediated by 5-HT1, 5-HT3 receptor and muscarinic cholinergic receptors in rat model of collagenase-induced osteoarthritis. BMC Complement Altern Med. 2016;16:212-22.

17. Zhang W, Nuki G, Moskowitz RW, Abramson S, Altman RD, Arden NK, et al. OARSI recommendations for the management of hip and knee osteoarthritis. Osteoarthr Cartil. 2010;18:476-99.

18. Temple AR, Benson GD, Zinsenheim JR, Schweinle JE. Multicenter, randomized, double-blind, active-controlled, parallel-group trial of the longterm (6-12 months) safety of acetaminophen in adult patients with osteoarthritis. Clin Ther. 2006;28:222-35.

19. Emery CA, Roos EM, Verhagen E, Finch CF, Bennell KL, Story B, et al. OARS clinical trials recommendations: design and conduct of clinical trials for primary prevention of osteoarthritis by joint injury prevention in sport and recreation. Osteoarthr Cartil. 2015;23:815-25.

20. Farzaei MH, Farzaei F, Gooshe M, Abbasabadi Z, Rezaei N, Abdolghaffari AH. Potentially effective natural drugs in treatment for the most common rheumatic disorder: osteoarthritis. Rheumatol Int. 2015;35:799-814

21. Iannitti T, Lodi D, Palmieri A. Intra-articular injections for the treatment of osteoarthritis. Drugs R D. 2011;11:13-27.

22. Park KK, Choi CH, Ha C-W, Lee MC. The effects of adherence to nonsteroidal anti-inflammatory drugs and factors influencing drug adherence in patients with knee osteoarthritis. J Korean Med Sci. 2016;31:795-816.

23. Massó González EL, Patrignani P, Tacconelli S, Rodríguez LAG. Variability among nonsteroidal antiinflammatory drugs in risk of upper gastrointestinal bleeding. Arthritis Rheum. 2010;62:1592-601.

24. McGettigan P, Henry D. Cardiovascular risk and inhibition of cyclooxygenase. JAMA. 2006;296:1633-44.

25. Hatori M, Kokubun S. Clinical evaluation of indomethacin-containing patches for osteoarthritis and extremity trauma. Curr Med Res Opin. 1997;13: $511-5$.

26. Sandberg O, Aspenberg P. Different effects of indomethacin on healing of shaft and metaphyseal fractures. Acta Orthop. 2015;86:243-7.

27. Terkeltaub RA, Schumacher HR, Carter JD, Baraf HS, Evans RR, Wang J, et al. Rilonacept in the treatment of acute gouty arthritis: a randomized, controlled clinical trial using indomethacin as the active comparator. Arthritis res. Ther. 2013;15:R25.

28. Kornasoff D, Maisenbacher J, Bowdler J, Raber A. The efficacy and tolerability of aceclofenac compared to indomethacin in patients with rheumatoid arthritis. Rheumatol Int. 1996:15:225-30.

29. Jung $Y B$, Roh $K$, Jung JA, Jung $K$, Yoo $H$, Cho YB, et al. Effect of SKI 306X, a new herbal anti-arthritic agent, in patients with osteoarthritis of the knee: a double-blind placebo controlled study. Am J Chin Med. 2001;29:485-91.

30. Lee CW, Park SM, Kim YS, Jegal KH, Lee JR, Cho IJ, et al. Biomolecular evidence of anti-inflammatory effects by Clematis mandshurica Ruprecht root extract in rodent cells. J Ethnopharmacol. 2014:155:1141-55.
31. Suh SJ, Kim KS, Lee SD, Lee CH, Choi HS, Jin U-H, et al. Effects and mechanisms of Clematis mandshurica maxim. As a dual inhibitor of proinflammatory cytokines on adjuvant arthritis in rats. Environ Toxicol Pharmacol. 2006:22:205-12.

32. Varas A, Valencia J, Lavocat F, Martínez VG, Thiam NN, Hidalgo L, et al. Blockade of bone morphogenetic protein signaling potentiates the proinflammatory phenotype induced by interleukin-17 and tumor necrosis factor-a combination in rheumatoid synoviocytes. Arthritis Res Ther. 2015; 17:192-201.

33. Hulejová H, Barešová V, Klézl Z, Polanská M, Adam M, Šenolt L. Increased level of cytokines and matrix metalloproteinases in osteoarthritic subchondral bone. Cytokine. 2007;38:151-6.

34. Valdes AM, Arden NK, Tamm A, Kisand K, Doherty S, Pola E, et al. A metaanalysis of interleukin-6 promoter polymorphisms on risk of hip and knee osteoarthritis. Osteoarthr Cartil. 2010;18:699-704.

35. Yuan PW, Liu DY, Chu XD, Hao YQ, Zhu C, Qu Q. Effects of preventive Administration of Juanbi Capsules on TNF-a IL-1 and IL-6 contents of joint fluid in the rabbit with knee osteoarthritis. J Tradit Chin Med. 2010;30:254-8.

36. Lee SH, Kwon KD, Lee SW, Ahn HS. Acetabular degeneration in osteonecrosis of the femoral head. J Korean Orthop Assoc. 2004;39:239-46.

37. Nieuwenhuizen L, Roosendaal G, Mastbergen SC, Coeleveld K, Biesma DH, Lafeber FPJG, et al. Antiplasmin, but not amiloride, prevents synovitis and cartilage damage following hemarthrosis in hemophilic mice. J Thromb Haemost. 2014;12:237-45.

38. Tiraloche G, Girard C, Chouinard L, Sampalis J, Moquin L, lonescu M, et al. Effect of oral glucosamine on cartilage degradation in a rabbit model of osteoarthritis. Arthritis Rheum. 2005;52:1118-28.

\section{Submit your next manuscript to BioMed Central and we will help you at every step:}

- We accept pre-submission inquiries

- Our selector tool helps you to find the most relevant journal

- We provide round the clock customer support

- Convenient online submission

- Thorough peer review

- Inclusion in PubMed and all major indexing services

- Maximum visibility for your research

Submit your manuscript at www.biomedcentral.com/submit
Biomed Central 